OPEN ACCESS

Edited by:

Bin Zhang,

South China University of

Technology, China

Reviewed by:

Ryszard Amarowicz,

Institute of Animal Reproduction and

Food Research (PAS), Poland

Man Li,

Qingdao Agricultural University, China

${ }^{*}$ Correspondence:

Lingyan Kong

Ikong@ches.ua.edu

tThese authors have contributed equally to this work and share first

authorship

Specialty section:

This article was submitted to

Food Chemistry,

a section of the journal

Frontiers in Nutrition

Received: 13 August 2021

Accepted: 26 October 2021

Published: 26 November 2021

Citation:

Guo J, Gutierrez A, Tan L and Kong L

(2021) Inhibitory Effect of Ascorbic

Acid on in vitro Enzymatic Digestion of

Raw and Cooked Starches.

Front. Nutr. 8:758367.

doi: 10.3389/fnut.2021.758367

\section{Inhibitory Effect of Ascorbic Acid on in vitro Enzymatic Digestion of Raw and Cooked Starches}

\author{
Jiayue Guo ${ }^{1 \dagger}$, Alyssa Gutierrez ${ }^{2 \dagger}$, Libo Tan ${ }^{1}$ and Lingyan Kong ${ }^{1 *}$ \\ ${ }^{1}$ Department of Human Nutrition and Hospitality Management, The University of Alabama, Tuscaloosa, AL, United States, \\ ${ }^{2}$ Department of Biological Sciences, The University of Alabama, Tuscaloosa, AL, United States
}

Ascorbic acid, also known as vitamin C, was previously reported to inhibit the activity of pancreatic $\alpha$-amylase, the primary digestive enzyme for starch. A major implication of such inhibition is a slowed rate of starch digestion into glucose, which thereby reduces postprandial hyperglycemia. The aim of this study was to explore the inhibitory effects of ascorbic acid at various concentrations on the in vitro digestion of high amylose maize starch (HAMS) and potato starch (PS) in both raw and cooked conditions. Resistant starch (RS) content, defined as the starch that remained after $4 \mathrm{~h}$ of simulated in vitro enzymatic digestion, was measured for the starch samples. Upon the addition of ascorbic acid, the RS contents increased in both raw and cooked starches. Cooking significantly reduced the RS contents as compared to raw starches, and less increase in RS was observed with the addition of ascorbic acid. The inhibitory effect of ascorbic acid on the digestion of raw starches showed a dose-dependent trend until it reached the maximum extent of inhibition. At the concentrations of 12.5 and $18.75 \mathrm{mg} / \mathrm{mL}$, ascorbic acid exhibited the most potent inhibitory effect on the in vitro starch digestion in raw and cooked conditions, respectively. Overall, our results strongly indicate that ascorbic acid may function as a glycemic modulatory agent beyond other important functions, and its effects persist upon cooking with certain concentrations applied.

Keywords: high amylose maize starch, potato starch, ascorbic acid, cooking, simulated in vitro digestion

\section{INTRODUCTION}

As one of the most common chronic diseases worldwide, diabetes mellitus is a major risk factor for cardiovascular diseases and is associated with an increased rate of morbidity and mortality (1). It is characterized by chronic hyperglycemia, which involves many alterations at the vascular tissue that accelerates the pathogenesis of diabetic complications (2). Among the determinants for glucose metabolism, diet plays an important role in the development of hyperglycemia, as excessive ingestion of calorie-dense and easily digestible foods can cause abnormal spikes in postprandial blood glucose level $(3,4)$. Since starch is the primary energy source in human diet, retarding starch digestion and glucose absorption could serve as an effective way for the prevention and treatment of hyperglycemia and related metabolic diseases (5-7). Accordingly, some antidiabetic drugs, such as acarbose, have been used to retard starch digestion through inhibiting the activity of digestive enzymes (8). Nevertheless, some drugs are reported to have certain side effects, such as hepatotoxicity, gastrointestinal disturbances and diarrhea (9). Therefore, food-based strategies are of great interest as they may have less side-effects and carry additional health benefits. As a group 
of dietary compounds with beneficial health effects, phenolic compounds have been proposed as natural inhibitors of human digestive enzymes, including $\alpha$-amylase, $\alpha$-glucosidase, and lipase, etc. One of the well-known compounds is ascorbic acid, which has demonstrated the ability to inhibit starch digestive enzymes $(10,11)$ and therefore may function as a glycemic modulatory agent.

Ascorbic acid, commonly known as vitamin C, is a watersoluble micronutrient essential for the proper functioning of the body. It can be found in many foods, particularly fruits and vegetables such as citrus fruit, broccoli, and spinach. It is required for the formation and maintenance of connective tissues, and serves as a potent antioxidant which protects the body from harmful free radicals (12). Since it is watersoluble, excess ascorbic acid is easily excreted in urine and rarely accumulates to toxic levels. Such property makes the utilization of ascorbic acid advantageous over medications that may cause adverse symptoms at high doses. Beyond being required for the aforementioned essential metabolic activities, ascorbic acid has also been shown to inhibit the activity of pancreatic $\alpha$-amylase, a digestive enzyme that plays the major role in breaking down starch into glucose, via noncompetitive antagonism (11). Although the mechanism of such enzymatic inhibition was well-investigated, the effect of ascorbic acid was only examined in an $\alpha$-amylase assay over the brief course of $30 \mathrm{~min}$ (11), which is only a fraction of the total digestive time of starch in the small intestinal tract. Moreover, few studies have evaluated the inhibitory effects of ascorbic acid with various concentrations using simulated in vitro digestion assays, therefore raising concern regarding the dose responses and how this may translate to reallife applications.

The objective of this study was to further investigate the inhibitory effect of ascorbic acid on starch digestion as well as to explore its general applicability as a glycemic modulatory agent. To simulate the digestion process in the small intestine, mixtures of starch and ascorbic acid were subjected to $4 \mathrm{~h}$ of in vitro digestion. The amount of starch that remained undigested after the 4 -h period was defined as the resistant starch (RS) content (13). Different concentrations of ascorbic acid were used to determine whether the inhibitory effect is dose dependent. In addition, starches were subjected to cooking prior to digestion to evaluate the practicality of ascorbic acid as a potential glycemic modulatory agent.

\section{MATERIALS AND METHODS}

\section{Materials}

High amylose maize starch (HAMS, Hylon VII) was kindly provided by Ingredion (Bridgewater, NJ, USA). Potato starch (PS, S2004) and ascorbic acid were purchased from Sigma-Aldrich Inc. (St. Louis, MO, USA). Digestible starch and resistant starch assay kit (K-DSTRS) was obtained from Megazyme (Wicklow, Ireland). Ethanol was purchased from VWR International (Radnor, PA, USA).

\section{In vitro Digestion}

The in vitro starch digestion was conducted according to the method by the Megazyme resistant starch assay kit with slight modifications. The enzyme solution, containing pancreatic $\alpha$-amylase and $\alpha$-amyloglucosidase $(0.8$ and $0.34 \mathrm{KU} / \mathrm{mL}$, respectively), was prepared immediately before use. Cooked starch was prepared by boiling starch in a $100^{\circ} \mathrm{C}$ water bath for $20 \mathrm{~min}$ and allowed to cool to room temperature $\left(20^{\circ} \mathrm{C}\right)$ before in vitro digestion. Starch $(100 \mathrm{mg})$ alone and starch mixed with $12.5,25,50$, or $75 \mathrm{mg}$ of ascorbic acid were weighed into $20 \mathrm{~mL}$ round-bottom test tubes. A test tube with no starch and inhibitors was used as the blank. An amount of $3.5 \mathrm{~mL}$ of $50 \mathrm{mM}$ sodium maleate buffer was added into each tube, and the reaction suspensions were mixed and placed in a water bath at $37^{\circ} \mathrm{C}$ for $5 \mathrm{~min}$ to equilibrate. Enzyme solution $(0.5 \mathrm{~mL})$ was added into each tube at a certain time interval with accurate timing for further sampling. The test tubes were capped and placed into a shaking water bath at $37^{\circ} \mathrm{C}$ and 170 strokes per min. After incubating for $4 \mathrm{~h}, 4.0 \mathrm{~mL}$ of $95 \%(\mathrm{v} / \mathrm{v})$ ethanol was added to each tube and was mixed vigorously. The samples were centrifuged $(3,600 \mathrm{~g}, 10 \mathrm{~min})$ and the supernatant was decanted. The pellet was resuspended in $8 \mathrm{~mL}$ of $50 \%(\mathrm{v} / \mathrm{v})$ ethanol and vigorously mixed. The centrifugation, washing, and decanting steps were then repeated twice, and the remaining pellet was used for the resistant starch measurements.

\section{Resistant Starch Content}

The RS content in starch samples was determined following the resistant starch assay procedure using the digestible starch and resistant starch assay kit (K-DSTRS) (14), with slight modifications. The remaining pellet obtained from the last step was resuspended in $2 \mathrm{~mL}$ of cold $1.7 \mathrm{M} \mathrm{NaOH}$ by stirring for $20 \mathrm{~min}$ in an ice bath, and then added with $8 \mathrm{~mL}$ of $1.0 \mathrm{M}$ sodium acetate buffer and $0.1 \mathrm{~mL}$ of $\alpha$-amyloglucosidase $(3,300 \mathrm{U} / \mathrm{mL})$. The tubes were mixed well and placed into a $50^{\circ} \mathrm{C}$ water bath for $30 \mathrm{~min}$. For samples containing $>10 \%$ RS content, the contents of the tubes were transferred and volumes adjusted to $100 \mathrm{~mL}$ in a volumetric flask with water. Aliquots of the diluted solutions were centrifuged (17,900 g, $5 \mathrm{~min}$ ). For samples containing $<10 \%$ RS content, aliquots of solutions (no dilution) were centrifuged directly $(17,900 \mathrm{~g}, 5 \mathrm{~min})$. In duplicates, aliquots of $30 \mu \mathrm{L}$ from the diluted or undiluted supernatants were measured for the glucose concentrations using the glucose oxidase-peroxidase (GOPOD) method (14). The reagent blank was prepared using $30 \mu \mathrm{L}$ of $100 \mathrm{mM}$ sodium acetate buffer, and the glucose standard was prepared in duplicates using $30 \mu \mathrm{L}$ of D-glucose $(1 \mathrm{mg} / \mathrm{mL})$. The absorbance was measured at $510 \mathrm{~nm}$ against the reagent blank. The RS contents were obtained using the appropriate MegaCalc ${ }^{\mathrm{TM}}$ Excel $^{\circledR}$ Calculator.

\section{Statistical Analysis}

All experiments were conducted in duplicates. Data were analyzed by one-way analysis of variance (one-way ANOVA) followed by Tukey's multiple comparison test using the OriginPro software (OriginLab, Northampton, MA, USA). The letters $\mathrm{a}, \mathrm{b}, \mathrm{c}$, and $\mathrm{d}$ indicate statistically significant differences, $p<0.05(a>b>c>d)$. 


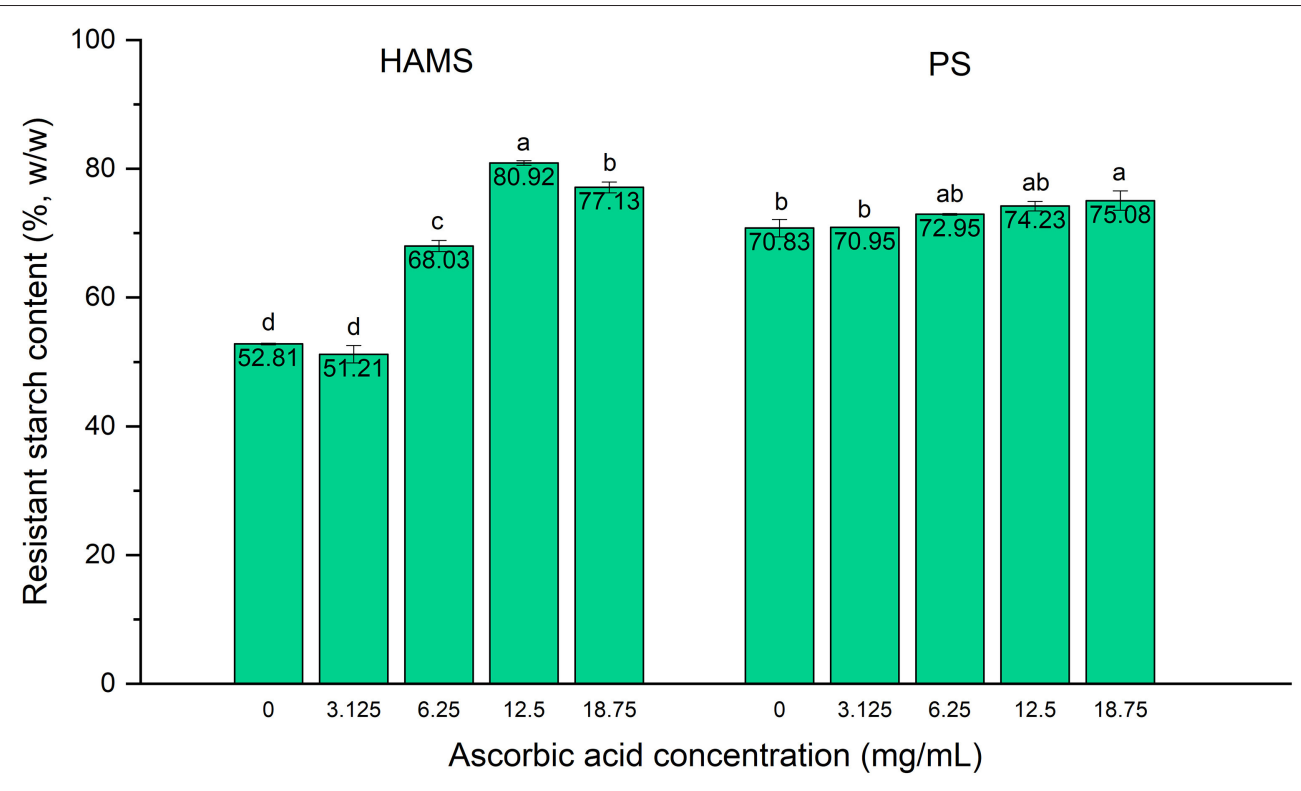

FIGURE 1 | Resistant starch contents in high amylose maize starch (HAMS) and potato starch (PS), presented as a proportion of total starch, with the presence of ascorbic acid at various concentrations. Error bars show standard deviation; $n=2$. Significant differences among treatments of differing ascorbic acid concentrations are denoted by different letters $(a>b>c>d, p<0.05)$.

\section{RESULTS AND DISCUSSION}

\section{Dose-Dependent Effect of Ascorbic Acid on Inhibiting Raw Starch Digestion}

The potential inhibitory effect of ascorbic acid on the in vitro starch digestion rate was first explored on two starches (HAMS and PS) in their raw form. To better understand its applicability as an inhibitor of starch digestion, four different concentrations $(3.125,6.25,12.5$, and $18.75 \mathrm{mg} / \mathrm{mL})$ were used to determine the most effective level of ascorbic acid that could retard starch digestion to the greatest extent. As shown in Figure 1, the RS content of raw HAMS was $52.81 \%$, which is very close to previous findings in HAMS, e.g., the reported $46.9 \%$ in Hi-Maize 260 starch (15), and the reference value, $47.4 \%$ in HAMS Hylon VII, provided by Megazyme (14). Upon the addition of ascorbic acid, the RS content in HAMS exhibited significant increase $(p<0.05)$ in response to all tested concentrations except for $3.125 \mathrm{mg} / \mathrm{mL}$. As the concentration of ascorbic acid increased up to 12.5 $\mathrm{mg} / \mathrm{mL}$, the RS content continued to increase, showing a dosedependent effect of ascorbic acid (Figure 1). However, increase of the ascorbic acid concentration to $18.75 \mathrm{mg} / \mathrm{mL}$ did not result in further increases in the RS content, demonstrating a saturation effect. Similar results were observed in PS, where a trend for dosedependent increase in the RS content was visible as ascorbic acid concentration increased from 3.125 to $18.75 \mathrm{mg} / \mathrm{mL}$, although only the concentration of $18.75 \mathrm{mg} / \mathrm{mL}$ resulted in a statistically significant increase.

As previously mentioned, such inhibitory effect of ascorbic acid on starch digestion is primarily due to the inhibition against $\alpha$-amylases. The underlying mechanism could be owing to the hydroxyl groups present in the ascorbic acid molecules, which may be crucial in binding to pancreatic $\alpha$-amylase, leading to the inhibitory activity (11). When the concentration of ascorbic acid increases, multiple ascorbic acid molecules can contribute more hydroxyl groups in forming hydrogen bonds with amino acid residues in the $\alpha$-amylase binding sites, which may explain the dose-dependent inhibitory effect. Besides, the changed $\mathrm{pH}$ by ascorbic acid in the reactant was also considered for such inhibitory effect. We tested the $\mathrm{pH}$ values of the original maleate buffer and with the addition of ascorbic acid of different concentrations, and found that the $\mathrm{pH}$ of the buffer solution reduced from 6.01 to 5.33 (with $3.125 \mathrm{mg} / \mathrm{mL}$ ascorbic acid), 4.56 (with 6.25 $\mathrm{mg} / \mathrm{mL}$ ), 3.91 (with $12.5 \mathrm{mg} / \mathrm{mL}$ ), and 3.65 (with $25 \mathrm{mg} / \mathrm{mL}$ ) with increasing ascorbic acid concentrations, respectively. As the optimal $\mathrm{pH}$ for $\alpha$-amylase and amyloglucosidase activity was in the range of 4.5-7.2 and 4.2-5.5, respectively (17), addition of ascorbic acid with concentrations up to 12.5 $\mathrm{mg} / \mathrm{mL}$ would create an acidic environment with the $\mathrm{pH}$ out of the optimal ranges of the starch digestive enzymes, which is another possible mechanism underlying the inhibitory effect of ascorbic acid against starch digestion. Consequently, our findings suggest similar result as reported by Borah et al. (11), who found that the inhibitory effect of ascorbic acid against human pancreatic $\alpha$-amylase is comparable to that of the reference inhibitor, i.e., acarbose, marking its strong potential as a starch digestion inhibitor and glycemic response modulator.

In this study, four different doses of ascorbic acid were applied, which were $3.125,6.25,12.5$, and $18.75 \mathrm{mg} / \mathrm{mL}$. Such doses were selected based on our previous finding that the addition of $25 \mathrm{mg}$ of ascorbyl palmitate was effective enough 
to significantly increase the RS content in the same amount of starch $(100 \mathrm{mg}$ ) (16). Based on this, to test the inhibitory effect in response to dose, ascorbic acid with amounts of 12.5, 25, 50 , and $75 \mathrm{mg}$ were added to $100 \mathrm{mg}$ of starch prior to the in vitro digestion. As the total volume of the solvent was $4 \mathrm{~mL}$, the concentrations of the added ascorbic acid correspond to $3.125,6.25,12.5$, and $18.75 \mathrm{mg} / \mathrm{mL}$, respectively. In addition, such doses are practical and applicable to incorporate into daily diet without causing any toxic effect. The absorption of ascorbic acid in small intestine is tightly regulated and peaks at $\sim 162$ $\mathrm{mg} /$ day (18). Since ascorbic acid is water-soluble and is easily excreted in urine, it rarely accumulates to toxic levels in tissues and plasma. At high intakes, the most common symptoms are gastrointestinal issues resulting from the osmotic effect of high concentrations of unabsorbed ascorbic acid, such as diarrhea and nausea (18). In the small intestinal tract, the total fluid volume reaches a maximum of $\sim 94 \mathrm{~mL}$ (19). Using the optimal concentration of ascorbic acid at $12.5 \mathrm{mg} / \mathrm{mL}$, the amount of ascorbic acid required $(\sim 1,175 \mathrm{mg})$ to saturate the peak fluid volume is still substantially below the tolerable upper intake level of ascorbic acid for adults at 2,000 mg/day (18). Even after compensating for ascorbic acid that is ultimately absorbed in the small intestine, the amount of ascorbic acid required to reach the maximum inhibitory activity is unlikely to be toxic in human applications. Meanwhile, it is practical to achieve such optimal dose of vitamin $\mathrm{C}$ via daily dietary intake or supplementation. For dietary sources, one serving of fruit or vegetable could contain up to $400 \mathrm{mg}$ of vitamin $\mathrm{C}$, and supplements typically contain 100-2,000 mg per capsule. By applying such dietary strategy, the recommended dietary allowance (RDA) of vitamin C could also be easily achieved, which is $90 \mathrm{mg} /$ day for adult men and 75 $\mathrm{mg}$ /day for adult women (20). This again suggests the practicality and applicability of ascorbic acid as a dietary strategy for the prevention and treatment of hyperglycemia.

\section{Effect of Cooking}

Starch digestibility is dependent on the activity of digestive enzymes as well as the characteristics of the starch itself, such as the botanical source and degree of gelatinization (21). Although the above results have confirmed the inhibitory effect of ascorbic acid on the in vitro digestion of raw starches, it is important to verify that such effect is present in forms of starch that would realistically be consumed in human diet, i.e., subjected to cooking. Accordingly, based on the results obtained from raw starches, we further studied the effects of ascorbic acid with concentrations of 3.125 and $12.5 \mathrm{mg} / \mathrm{mL}$ on the digestion of cooked HAMS and PS (Figure 2).

Heating starch in the presence of water, i.e., cooking, results in gelatinization of starch. This process causes the disruption and swelling of starch granules, leaching of amylose into the water, and the loss of molecular order in amylopectin. Upon heating, water enters the amorphous regions and then disrupts the crystalline regions. These changes are accompanied by swelling of the granules, which will contribute to the eventual collapse of the granules to form a paste if the water content is high enough (22). Consequently, the starch is more readily accessible and susceptible to enzymatic digestion (23). This outcome is reflected

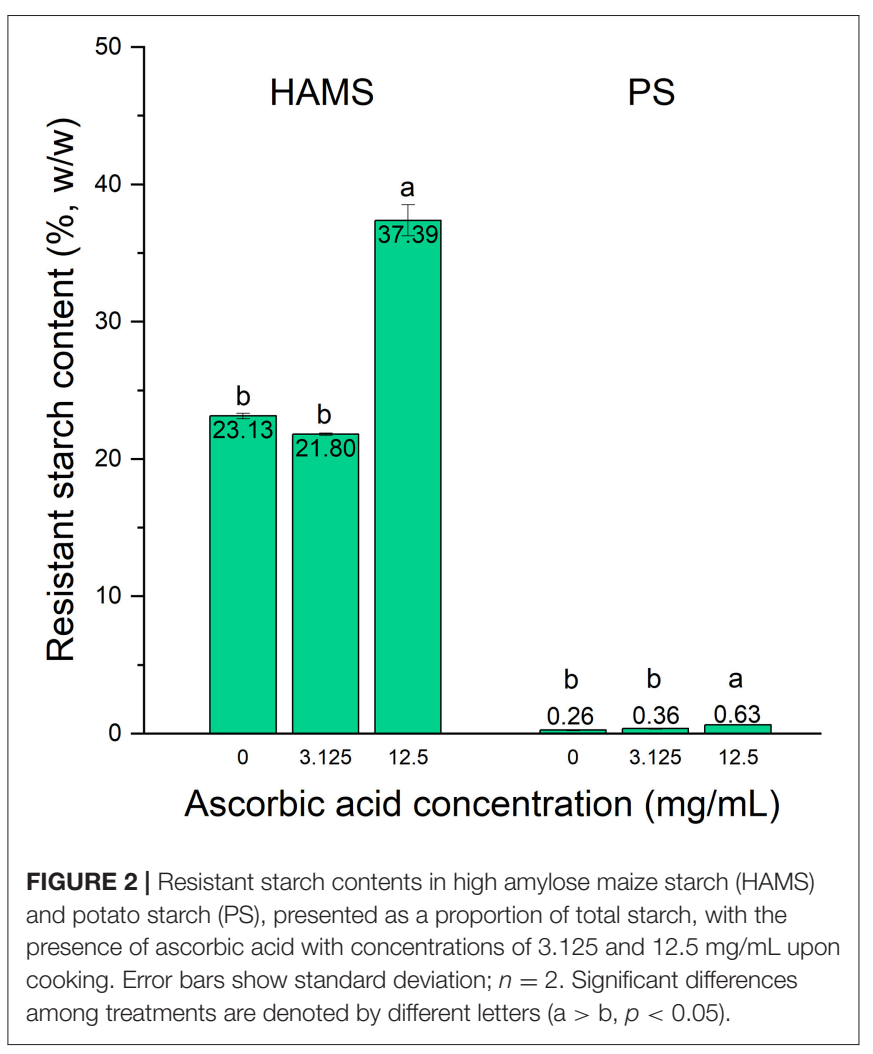

by the relatively low RS content for the cooked starches, which is 23.13 and $0.26 \%$ for HAMS and PS, respectively, as compared to 52.81 and $70.83 \%$ in their raw forms. PS was more susceptible to gelatinization as compared to HAMS, which could be due to its lower gelatinization temperature, which is $59-68^{\circ} \mathrm{C}$ as compared to $125^{\circ} \mathrm{C}$ for $\operatorname{HAMS}(24,25)$. This result is consistent with other reports that the gelatinization of starch results in significant reductions of RS contents in canna, rice, and potato starches $(5,26,27)$.

Similar to the results observed for raw starches, ascorbic acid with a concentration of $3.125 \mathrm{mg} / \mathrm{mL}$ did not present any inhibitory effect on starch digestion, while $12.5 \mathrm{mg} / \mathrm{mL}$ of ascorbic acid resulted in significant increases in the RS contents in both HAMS and PS, although the effects were rather modest as compared to those in raw starches. For cooked HAMS, the RS content was increased to $37.39 \%(p<0.05)$ in the presence of ascorbic acid, whereas that of cooked PS was only increased to $0.63 \%(p<0.05)$. Such modest enhancement could be explained by the destruction in the starch molecular packing order caused by gelatinization, which could not be recovered or altered in any way by the presence of ascorbic acid. This also indicates that ascorbic acid can only act on the digestive enzymes, but not the substrate, to resist enzymatic hydrolysis (11). Therefore, as the susceptibility of starch granules to enzymatic hydrolysis increases after cooking, fewer digestive enzymes are needed, resulting in the less potent inhibitory effect of ascorbic acid against starch digestion as compared to what was observed in raw starches. In addition, the modest effect could be attributed to the high 
moisture content of the cooking condition. After gelatinization, starch may undergo a process called retrogradation, in which the disordered amylose and amylopectin molecules re-associate into crystallized structures. Retrograded starch contributes to increased RS contents because the more ordered arrangement results in slower enzymatic digestion (28). The storage of gelatinized starch facilitates this process, but the water content plays a major role in the extent to which the starch retrogrades. More specifically, retrogradation for maize starch only occurs when the water content is between 20 and 90\% (28). In our study, the moisture content of the gelatinized starch was $\sim 97 \%$, as the starches were suspended in the buffer solutions while being cooked, thereby rendering retrogradation unlikely. This could possibly lead to the low RS content after cooking even with the addition of ascorbic acid.

\section{CONCLUSION}

The inhibitory effect of ascorbic acid on simulated in vitro starch digestion, as evidenced by increased contents of RS, was demonstrated in this study. Our results indicated the dosedependent response of such inhibitory effect, and that the optimal level of ascorbic acid is practical and achievable via daily food or supplement intake and unlikely to result in toxicity in human body. Furthermore, in the tested cooking condition, the RS contents were significantly increased in the presence of ascorbic acid, although to a modest extent. This evidence suggests that ascorbic acid can be broadly applied to realistic starch systems. The inhibitory ability was most potent in the case of raw starch, and the slighter impact on cooked starch is likely the result of gelatinization with a high water content. Thus, future studies illustrating the inhibitory effect of ascorbic acid on starch

\section{REFERENCES}

1. Marcovecchio ML, Lucantoni M, Chiarelli F. Role of chronic and acute hyperglycemia in the development of diabetes complications. Diabetes Technol Ther. (2011) 13:389-94. doi: 10.1089/dia.2010.0146

2. Aronson D. Hyperglycemia and the pathobiology of diabetic complications. Adv Cardiol. (2008) 45:1-16. doi: 10.1159/000115118

3. Chiu C-J, Taylor A. Dietary hyperglycemia, glycemic index and metabolic retinal diseases. Prog Retinal Eye Res. (2011) 30:18-53. doi: 10.1016/j.preteyeres.2010.09.001

4. Tiwari AK, Reddy KS, Radhakrishnan J, Kumar DA, Zehra A, Agawane $\mathrm{SB}$, et al. Influence of antioxidant rich fresh vegetable juices on starch induced postprandial hyperglycemia in rats. Food Funct. (2011) 2:521-8. doi: 10.1039/c1fo10093a

5. Gutierrez ASA, Guo J, Feng J, Tan L, Kong L. Inhibition of starch digestion by gallic acid and alkyl gallates. Food Hydrocoll. (2020) 102:105603. doi: 10.1016/j.foodhyd.2019.105603

6. Sun L, Miao M. Dietary polyphenols modulate starch digestion and glycaemic level: a review. Crit Rev Food Sci Nutr. (2020) 60:541-55. doi: 10.1080/10408398.2018.1544883

7. Guo J, Tan L, Kong L. Impact of dietary intake of resistant starch on obesity and associated metabolic profiles in human: a systematic review of the literature. Critic Rev Food Sci Nutr. (2021) 61:889-905. doi: 10.1080/10408398.2020.1747391

8. Clissold SP, Edwards C. Acarbose. Drugs. (1988) 35:214-43. doi: 10.2165/00003495-198835030-00003 cooked in relatively drier conditions are warranted to determine the effects of retrogradation on starch digestibility. Given that ascorbic acid has already been established as an essential nutrient, this study supports its further utilization as a promising agent in glycemic modulation.

\section{DATA AVAILABILITY STATEMENT}

The original contributions presented in the study are included in the article/supplementary material, further inquiries can be directed to the corresponding author.

\section{AUTHOR CONTRIBUTIONS}

AG: investigation, formal analysis, visualization, and writingoriginal draft. JG: investigation, formal analysis, visualization, and writing-review and editing. LT: conceptualization, formal analysis, and writing-review and editing. LK: conceptualization, supervision, formal analysis, visualization, and writing-review and editing. All authors contributed to the article and approved the submitted version.

\section{ACKNOWLEDGMENTS}

This project is funded by the USDA National Institute for Food and Agriculture, Agriculture and Food Research Initiative Program, Competitive Grants Program award from the Improving Food Quality (A1361) program FY 2018 as grant \# 2018-67017-27558. The Emerging Scholars Program of the University of Alabama is acknowledged for sponsoring AG as an undergraduate researcher.

9. Filippatos TD, Derdemezis CS, Gazi IF, Nakou ES, Mikhailidis DP, Elisaf MS. Orlistat-associated adverse effects and drug interactions: a critical review. Drug Saf. (2008) 31:53-65. doi: 10.2165/00002018-200831010-00005

10. Abell AD, Ratcliffe MJ, Gerrard J. Ascorbic acid-based inhibitors of $\alpha$-amylases. Bioorg Med Chem Lett. (1998) 8:1703-6. doi: 10.1016/S0960-894X(98)00298-4

11. Borah PK, Sarkar A, Duary RK. Water-soluble vitamins for controlling starch digestion: conformational scrambling and inhibition mechanism of human pancreatic $\alpha$-amylase by ascorbic acid and folic acid. Food Chem. (2019) 288:395-404. doi: 10.1016/j.foodchem.2019.03.022

12. Bendich A, Langseth L. The health effects of vitamin C supplementation: a review. J Am Coll Nutr. (1995) 14:124-36. doi: 10.1080/07315724.1995.10718484

13. McCleary BV, McLoughlin C, Charmier LMJ, McGeough P. Measurement of available carbohydrates, digestible, and resistant starch in food ingredients and products. Cereal Chem. (2020) 97:114-37. doi: 10.1002/cche. 10208

14. Megazyme. Resistant Starch Assay Procedure KRAPRS. (2019). Available online at: https://www.megazyme.com/resistant-starch-assay-kit-rapid (accessed Oct 22, 2021)

15. Foschia M, Beraldo P, Peressini D. Evaluation of the physicochemical properties of gluten-free pasta enriched with resistant starch. J Sci Food Agric. (2017) 97:572-7. doi: 10.1002/jsfa.7766

16. Guo J, Kong L. Inhibition of in vitro starch digestion by ascorbyl palmitate and its inclusion complex with starch. Food Hydrocoll. (2021) 121:107032. doi: 10.1016/j.foodhyd.2021.107032 
17. Clementi F, Rossi J. Alpha-amylase and glucoamylase production by Schwanniomyces castellii. Antonie Van Leeuwenhoek. (1986) 52:343-52. doi: $10.1007 / \mathrm{BF} 00428645$

18. Health NIo. Vitamin C Fact Sheet for Health Professionals. [Fact Sheet] (2019).

19. Mudie DM, Murray K, Hoad CL, Pritchard SE, Garnett MC, Amidon GL, et al. Quantification of gastrointestinal liquid volumes and distribution following a $240 \mathrm{~mL}$ dose of water in the fasted state. Mol Pharm. (2014) 11:3039-47. doi: $10.1021 / \mathrm{mp} 500210 \mathrm{c}$

20. Institute of Medicine (US) Panel on Dietary Antioxidants and Related Compounds. Dietary Reference Intakes for Vitamin C, Vitamin E, Selenium, and Carotenoids. Washington, DC: National Academies Press (2000).

21. Rooney L, Pflugfelder R. Factors affecting starch digestibility with special emphasis on sorghum and corn. J Anim Sci. (1986) 63:1607-23. doi: $10.2527 /$ jas $1986.6351607 x$

22. Wang S, Copeland L. Molecular disassembly of starch granules during gelatinization and its effect on starch digestibility: a review. Food Funct. (2013) 4:1564-80. doi: 10.1039/c3fo60258c

23. Ratnayake WS, Jackson DS. Starch gelatinization. Adv Food Nutr Res. (2008) 55:221-68. doi: 10.1016/S1043-4526(08)00405-1

24. Chen X, Guo L, Chen P, Xu Y, Hao H, Du X. Investigation of the high-amylose maize starch gelatinization behaviours in glycerol-water systems. J Cereal Sci. (2017) 77:135-40. doi: 10.1016/j.jcs.2017.08.012

25. Ding L, Huang Q, Li H, Wang Z, Fu X, Zhang B. Controlled gelatinization of potato parenchyma cells under excess water condition: structural and in vitro digestion properties of starch. Food Funct. (2019) 10:5312-22. doi: 10.1039/C9FO00928K

26. Juansang J, Puttanlek C, Rungsardthong V. Puncha-arnon S, Uttapap D. Effect of gelatinisation on slowly digestible starch and resistant starch of heat-moisture treated and chemically modified canna starches. Food Chem (2012) 131:500-7. doi: 10.1016/j.foodchem.2011.09.013

27. Eroglu EI, Buyuktuncer Z. The effect of various cooking methods on resistant starch content of foods. Nutr Food Sci. (2017) 47:522-33. doi: 10.1108/NFS-10-20 16-0154

28. Wang S, Li C, Copeland L, Niu Q, Wang S. Starch retrogradation: a comprehensive review. Compr Rev Food Sci Food Saf. (2015) 14:568-85. doi: 10.1111/1541-4337.12143

Conflict of Interest: The authors declare that the research was conducted in the absence of any commercial or financial relationships that could be construed as a potential conflict of interest.

Publisher's Note: All claims expressed in this article are solely those of the authors and do not necessarily represent those of their affiliated organizations, or those of the publisher, the editors and the reviewers. Any product that may be evaluated in this article, or claim that may be made by its manufacturer, is not guaranteed or endorsed by the publisher.

Copyright (c) 2021 Guo, Gutierrez, Tan and Kong. This is an open-access article distributed under the terms of the Creative Commons Attribution License (CC BY). The use, distribution or reproduction in other forums is permitted, provided the original author(s) and the copyright owner(s) are credited and that the original publication in this journal is cited, in accordance with accepted academic practice. No use, distribution or reproduction is permitted which does not comply with these terms. 